\title{
Absorbing Markov Chain Models to Determine Optimum Process Target Levels in Production Systems with Dual Correlated Quality Characteristics
}

\author{
Mohammad Saber Fallah Nezhad \\ Industrial Engineering \\ Yazd University, Yazd, Iran \\ Fallahnezhad@yazduni.ac.ir \\ Hasan Hosseini Nasab \\ Industrial Engineering \\ Yazd University, Yazd, Iran \\ hhn@yazduni.ac.ir
}

\begin{abstract}
For a manufacturing organization to compete effectively in the global marketplace, cutting costs and improving overall efficiency is essential. A single-stage production system with two independent quality characteristics and different costs associated with each quality characteristic that falls below a lower specification limit (scrap) or above an upper specification limit (rework) is presented in this paper. The amount of reworks and scraps are assumed to be depending on the process parameters such as process mean and standard deviation thus the expected total profit is significantly dependent on the process parameters. This paper develops a Markovian decision making model for determining the process means. Sensitivity analyzes is performed to validate, and a numerical example is given to illustrate the proposed model. The results showed that the optimal process means extremely effects on the quality characteristics' parameters.
\end{abstract}

Keywords: Markov Chain, Process Mean, Bi-variate Normal Distribution.

\section{Introduction}

A product in the manufacturing process usually should satisfy a set of specifications. One of the important parameters of a quality characteristic is the product target mean. The problem of selecting the optimal target means has been an impotent research area for many years. Determining the optimal target mean of a quality characteristic is financially important. Basically, if deviations in the two directions of quality characteristics have equal cost, then the optimal mean of the process is the middle point of the tolerance limits. However, when the deviation of a quality characteristic in one direction is more costly than in the opposite direction, the optimal mean of the process is not the middle point of the tolerance limits (Abbasi et al. 2006).

In a manufacturing process, the defective items are usually produced with finished goods. Rework process is necessary to convert those defectives into finished goods. As many systems are not perfect, some scrap is produced during this process of manufacturing and rework. These scraps and reworks can reduce profitability. Many statistical tools have been developed to maximize the potential profit for an item in production settings. 
Rahim and Al-Sultan (2000) considered the problem of simultaneously determining the optimal target mean and target variance for a process that might result in a reduction in variability and in the total cost of the production process.

Costa and Rahim (2001) presented an economic design of control charts with variable sample sizes, variable sampling intervals, and variable control limits. They constructed a cost model that involves the cost of false alarms, the cost of finding and eliminating the assignable cause, the cost associated with production in an out-of-control state, and the cost of sampling and testing. They assumed an exponential distribution to describe the length of time the process remains in control for applying the Markov chain approach for developing the cost function.

Pignatiello and Tsai (1988) suggested a method for using cost models for designing control charts when precise estimates of the cost model parameters are not available. Their control chart designs are not sensitive to the estimates of the various cost model parameters by incorporating a measure of the imprecision of the parameter estimates into the objective function. Tosirisuk (1990) obtained the optimal process parameter control limits and process adjustment intervals, which minimize the total quality cost of production. Khasawneh et al. (2008) considered dual quality characteristics and different costs associated with each quality characteristic that falls below a lower specification limit or above an upper specification limit.

\subsection{Background}

In a certain production process, where an item has two quality characteristics, if the value of both quality characteristics falls within the tolerance limits then the item is faultless and can be accepted. The item is considered as scrape if the value of one of its quality characteristics falls below a lower tolerance limit. Moreover, the item needs to rework, if the value of one or both quality characteristics fall above an upper tolerance limit. In such a system, if the process mean is set to a low level (near to lower tolerance limit), then the proportion of defective items' increases and therefore, the system experiences high scrapped items' cost. But, if the process mean is set to a high level (near to upper tolerance limit), then the proportion of items that needs rework increases and thus the system is faced with high rework cost. This justifies the determination of an optimum process mean (Fallahnezhad and Niaki, 2011).

Khasawneh et al. (2008) considered a production system that consists of a single machine and a single inspection station. They assumed that any product has two-quality characteristics. Each product is processed and its quality characteristics are examined at an inspection station. In their model, an item is reworked, if its performance associated with the quality characteristic of interest falls above an upper specification limit, scrapped if its performance falls below a lower specification limit, or accepted if its performance falls within the specification limits. They assumed that rework process follows a normal distribution function similar to initial production process therefore, its process mean is equal to initial production process. However, in many real cases, the 
rework process follows different process function that should be considered to reduce the total production costs. In this research, we assume that reworked items will be accepted with constant probabilities and each item may be accepted or rejected after the reworked process. In this case, the maximum number of reworked process for any item is single.

In this paper, a production process with two quality characteristics is considered. A Markovian model is developed where defective items consisting of scrap and rework are produced, detected, and discarded during the process of manufacturing. To optimum the expected profit, scraps and reworks costs are considered in the model which is discussed in section 2 . The optimum process means for two quality characteristics is determined in section 3. A sensitivity analysis is performed by varying the cost parameters, such as scrap cost, rework cost in section 4. A numerical example is provided in section 5.

\section{The proposed Model}

Assume that two quality characteristics $x$ and $y$ follow a bi-variate normal distribution as is shown in equation 1.

$$
f(x, y)=\frac{1}{2 \pi \sigma_{x} \sigma_{y} \sqrt{1-\rho^{2}}} e^{-\frac{1}{2\left(1-\rho^{2}\right)}\left(\left(\frac{x-\mu_{x}}{\sigma_{x}}\right)^{2}+\left(\frac{y-\mu_{y}}{\sigma_{y}}\right)^{2}-2 \rho\left(\frac{x-\mu_{x}}{\sigma_{x}}\right)\left(\frac{y-\mu_{y}}{\sigma_{y}}\right)\right)}
$$

The notations $U S L_{x}$ and $L_{S L}$ are the upper and lower specification limits for the characteristic $x$ and the notations $U_{S L}$ and $L_{S S}$ are the upper and lower specification limits for characteristic $y$. In a process, if a quality characteristic was less than its lower specification limit then the product is considered as scrape, and if it was more than the upper specification limit then the product needs to rework. Other notations are defined as:

$c_{x} \quad$ The cost of reworking characteristic $x$

$c_{y} \quad$ The cost of reworking characteristic $y$

c Cost of a scrapped item

pc Manufacturing process cost for each item

$p \quad$ The profit per item

For a single-stage production system with the following states:

State 1: An item is being processed by the production system

State 2: The characteristic $\mathrm{x}$ is being reworked.

State 3: The characteristic $y$ is being reworked.

State 4: The characteristic $x$ and $y$ are being reworked

State 5: An item is accepted to be finished work

State 6: An item is scrapped. 
The single-step transition probability matrix can be expressed as:

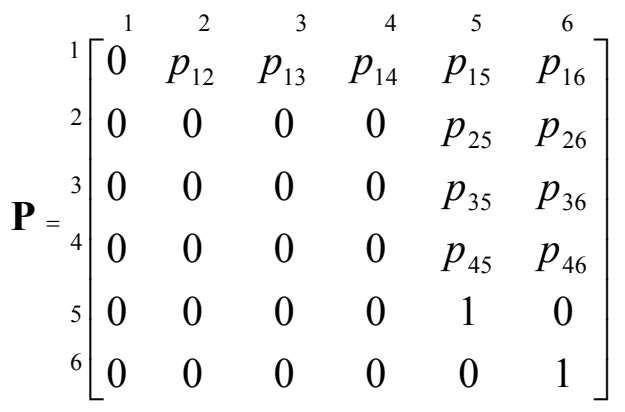

where

$p_{12} \quad$ The probability of reworking the characteristics $\mathrm{x}$

$p_{13} \quad$ The probability of reworking characteristics y

$p_{14} \quad$ The probability of reworking both characteristics $\mathrm{x}$ and $\mathrm{y}$

$p_{15} \quad$ The probability of accepting an item

$p_{16} \quad$ The probability of a scrap item

Moreover, for the characteristic $\mathrm{x}, p_{25}$ denotes the probabilities of accepting an item and $p_{26}$, scrapping an item after reworked. For characteristic $\mathrm{y}$ those are $p_{35}$ and $p_{36}$. Finally, $p_{45}$ and $p_{46}$ denote the probabilities of accepting and scrapping an item after its characteristic $\mathrm{x}$ and $\mathrm{y}$ are reworked.

Assuming that the quality characteristics of an item follow a bi-variate normal distribution, so that its probabilities can be expressed as:

$$
\begin{aligned}
& p_{12}=\operatorname{Pr}\left\{x>U S L_{x}, L S L_{y} \leq y \leq U S L_{y}\right\}=\int_{L S L_{y}}^{U S L_{y}} \int_{U S L_{x}}^{\infty} f(x, y) d x d y \\
& p_{13}=\operatorname{Pr}\left\{L S L_{x} \leq x \leq U S L_{x}, y>U S L_{y}\right\}=\int_{U S L_{y}}^{\infty} \int_{L S L_{x}}^{U S L_{x}} f(x, y) d x d y \\
& p_{14}=\operatorname{Pr}\left\{x>U S L_{x}, y>U S L_{y}\right\}=\int_{U S L_{y}}^{\infty} \int_{U S L_{x}}^{\infty} f(x, y) d x d y \\
& p_{15}=\operatorname{Pr}\left\{L S L_{x} \leq x \leq U S L_{x}, L S L_{y} \leq y \leq U S L_{y}\right\}=\int_{L S L_{y}}^{U S L_{y}} \int_{L S L_{x}}^{U S L_{x}} f(x, y) d x d y \\
& p_{16}=\operatorname{Pr}\left\{L S L_{x}>x \text { or } L S L_{y}>y\right\}=\int_{-\infty}^{L S L_{y}} \int_{-\infty}^{\infty} f(x, y) d x d y+ \\
& \int_{-\infty}^{\infty} \int_{-\infty}^{L S L_{x}} f(x, y) d x d y-\int_{-\infty}^{L S L_{y}} \int_{-\infty}^{L S L_{x}} f(x, y) d x d y
\end{aligned}
$$


To analyze the absorbing Markov chain, the transition matrix $T$ is rearranged to the matrix $P$ as follows:

$$
T=\left[\begin{array}{ll}
\mathrm{I} & \mathbf{O} \\
\mathbf{R} & \mathbf{Q}
\end{array}\right]
$$

Therefore,

$$
\mathbf{P}=5_{6}^{6}\left[\begin{array}{cccccc}
1^{5} & 0 & 0 & 0 & 0 & 0 \\
0 & 1 & 0 & 0 & 0 & 0 \\
p_{15} & p_{16} & 0 & p_{12} & p_{13} & p_{14} \\
p_{25} & p_{26} & 0 & 0 & 0 & 0 \\
p_{35} & p_{36} & 0 & 0 & 0 & 0 \\
p_{45} & p_{46} & 0 & 0 & 0 & 0
\end{array}\right]
$$

Fundamental matrix $\mathbf{M}$ is determined as follows:

$$
\mathbf{M}=(\mathbf{I}-\mathbf{Q})^{-1}=\left[\begin{array}{cccc}
1 & -p_{12} & -p_{13} & -p_{14} \\
0 & 1 & 0 & 0 \\
0 & 0 & 1 & 0 \\
0 & 0 & 0 & 1
\end{array}\right]^{-1}=\left[\begin{array}{cccc}
1 & p_{12} & p_{13} & p_{14} \\
0 & 1 & 0 & 0 \\
0 & 0 & 1 & 0 \\
0 & 0 & 0 & 1
\end{array}\right]
$$

The absorption probability matrix $\mathbf{F}$ is determined as follows (Bowling et al. 2004):

$$
\begin{aligned}
& \mathbf{F}=\mathbf{M} \times \mathbf{R}=\left[\begin{array}{cccc}
1 & p_{12} & p_{13} & p_{14} \\
0 & 1 & 0 & 0 \\
0 & 0 & 1 & 0 \\
0 & 0 & 0 & 1
\end{array}\right] \times\left[\begin{array}{cc}
p_{15} & p_{16} \\
p_{25} & p_{26} \\
p_{35} & p_{36} \\
p_{45} & p_{46}
\end{array}\right]= \\
& {\left[\begin{array}{cc}
p_{15}+p_{12} p_{25}+p_{13} p_{35}+p_{14} p_{45} & p_{16}+p_{12} p_{26}+p_{13} p_{36}+p_{14} p_{46} \\
p_{25} & p_{26} \\
p_{35} & p_{36} \\
p_{45} & p_{46}
\end{array}\right]}
\end{aligned}
$$

Where $f_{15}$ and $f_{16}$ are the probabilities of accepting and scrapping one item respectively.

Now, the expected profit per item can be defined as:

$E(P R)=f_{15} p-p c-p_{12} c_{x}-p_{13} c_{y}-p_{14}\left(c_{x}+c_{y}\right)-f_{16} c$ 
With substituting, $f_{15}$ the optimal value of $\mu_{x}, \mu_{y}$ that maximize the expected profit can be reached.

$$
\begin{aligned}
& E(P R)=\left(p_{15}+p_{12} p_{25}+p_{13} p_{35}+p_{14} p_{45}\right) p-p c-p_{12} c_{x}-p_{13} c_{y}- \\
& p_{14}\left(c_{x}+c_{y}\right)-\left(p_{16}+p_{12} p_{26}+p_{13} p_{36}+p_{14} p_{46}\right) c
\end{aligned}
$$

\section{Numerical Example}

Considering a single-stage production system with the following parameters:

$p=120, p c=45, c_{x}=15, c_{y}=12, c=14, \sigma_{x}=1, \sigma_{y}=1, \rho=0, L_{x}=8.0, L_{y}=13.0$, $U_{x}=12.0$ and $U_{y}=17.0$.

Furthermore, from the historical data, we have $p_{25}=0.9, p_{35}=0.9, p_{45}=0.8$. Using the search procedure by plotting $E(P R)$ as a function of $\mu_{x}, \mu_{y}$, it is concluded that the expected profit is maximized at $\mu_{x}=10.4, \mu_{y}=15.4$ with an amount of 69.88 . The optimal values of $\mu_{x}, \mu_{y}$ are determined by evaluating the value of objective function for different values of $\mu_{x}, \mu_{y}$.

The expected profit as a function of the process means $\left(\mu_{x}, \mu_{y}\right)$ is shown in figure 1.

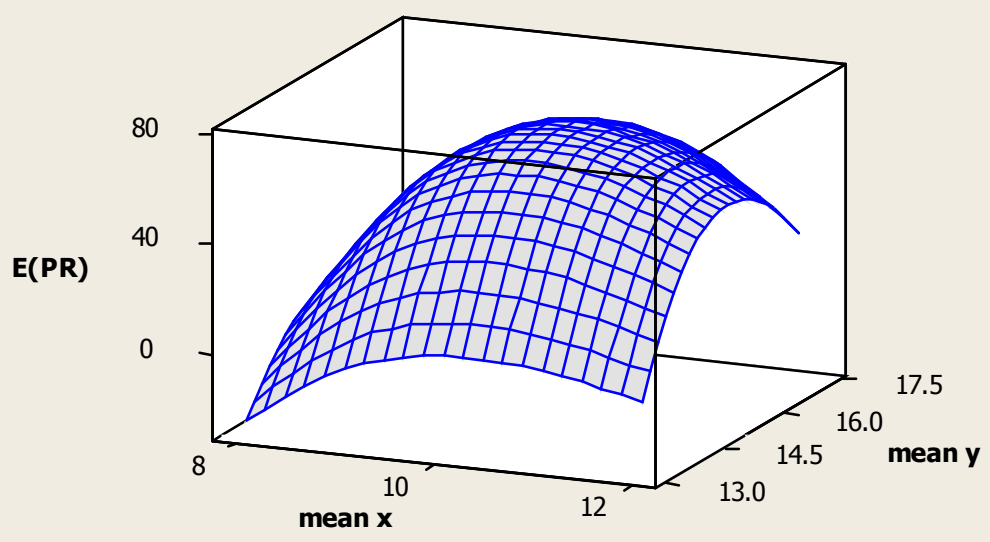

Figure 1: the expected profit as a function of the process means $\left(\mu_{x}, \mu_{y}\right)$ 
The expected profit is a concave function over the interval $\left(L_{x}=8, U_{x}=12\right)$, $\left(L_{y}=13, U_{y}=17\right)$. A sensitivity analysis is performed to analyze the possible impact of the rework and scrap costs on the optimal process means and the optimal expected profit. The effect of different reworked and scrap costs at each state are shown in the Table 1.

Table 1: The sensitivity results of different rework and scrap costs

\begin{tabular}{|c|c|c|c|c|c|}
\hline $\boldsymbol{c}_{\boldsymbol{x}}$ & $\boldsymbol{c}_{\boldsymbol{y}}$ & $\boldsymbol{c}$ & $\boldsymbol{E}(\boldsymbol{P R})$ & $\mu_{x}$ & $\mu_{y}$ \\
\hline 15 & 12 & 14 & 69.88 & 10.4 & 15.4 \\
\hline 1 & 12 & 14 & 70.74 & 10.6 & 15.4 \\
\hline 80 & 12 & 14 & 67.5 & 10.1 & 15.4 \\
\hline 15 & 1 & 14 & 70.58 & 10.35 & 15.55 \\
\hline 15 & 40 & 14 & 68.61 & 10.4 & 15.2 \\
\hline 15 & 12 & 1 & 70.24 & 10.35 & 15.4 \\
\hline 15 & 12 & 50 & 68.9 & 10.4 & 15.4 \\
\hline 15 & 12 & 120 & 67 & 10.4 & 15.4 \\
\hline 15 & 12 & 100 & 67.54 & 10.4 & 15.4 \\
\hline 200 & 12 & 14 & 64.84 & 9.80 & 15.40 \\
\hline 15 & 200 & 14 & 64.68 & 10.40 & 14.80 \\
\hline
\end{tabular}

Table 1 indicates that, by increasing the value of $c_{x}$, the optimum value of $\mu_{x}$ decreases, but it does not affect on the optimum value of $\mu_{y}$ also by increasing the value of $c_{y}$, the optimum value of $\mu_{y}$ decreases but simultaneously the optimum value of $\mu_{x}$ slightly increases, Also changing the value of $c$ changes the optimum value of $\mu_{x}, \mu_{y}$.

\section{Conclusion}

In this paper, a production process with two quality characteristics has been considered. A Markovian model is developed for determining the expected profit by considering processing, scrap, and rework costs. One numerical example was provided to illustrate the applications of the proposed models. The results showed that the rework and scrap cost for both quality characteristics extremely effects on the optimal process means.

\section{References}

1. Abbasi, B., Niaki, S.T.A., and Arkat J.(2006). Optimum Target Value for Multivariate Processes with Unequal Non-Conforming Costs, Journal of Industrial Engineering International, 2, 3, 1-12.

2. Bowling, S.R., M.T., Khasawneh, S., and Kaewkuekool, Cho, B.R. (2004). A Markovian approach to determining optimum process target levels for a multi-stage serial production system, European Journal of Operational Research, 159, 636-650. 
3. Costa, F.B., and Rahim, M.A. (2001). Economic design of $X$ charts with variable parameters: The Markov chain approach, Journal of Applied Statistics, 28, 7, $875-885$.

4. Fallahnezhad, M.S., and Niaki S.T.A. Absorbing Markov Chain Models to Determine Optimum Process Target Levels in Production Systems with Rework and Scrapping, To appear in Journal of Industrial Engineering, Qazvin Islamic Azad University.

5. Khasawneh, M.T., Bowling, S.R., and Cho, B.R. (2008). A Markovian Approach to Determining Process Means with Dual Quality Characteristics, J SystSciSystEng 17,1, 066-085.

6. Pignatiello, J.J., and Tsai, A. (1988).Optimal Economic Design of XControl Charts When Cost Model Parameters are Not Precisely Known IIE Transactions, 20, 1, $103-110$.

7. Rahim, M.A., and Al-Sultan, K.S. (2000). Joint determination of the optimum target mean and variance of a process, Journal of Quality in Maintenance Engineering, 6, 3,192 - 199.

8. Tosirisuk, P.(1990). Economic design of process parameter control limits and process adjustment intervals for continuous production processes, Computers \& Industrial Engineering, 19, 1-4, 263-266. 